\title{
The zCOSMOS redshift survey: the three-dimensional classification cube and bimodality in galaxy physical properties ${ }^{\star}$
}

\author{
M. Mignoli ${ }^{1}$, G. Zamorani ${ }^{1}$, M. Scodeggio ${ }^{2}$, A. Cimatti ${ }^{3}$, C. Halliday ${ }^{4, \star \star}$, S. J. Lilly ${ }^{5}$, L. Pozzetti ${ }^{1}$, D. Vergani ${ }^{1,2}$, \\ C. M. Carollo ${ }^{5}$, T. Contini ${ }^{6}$, O. Le Févre ${ }^{7}$, V. Mainieri ${ }^{8}$, A. Renzini ${ }^{9}$, S. Bardelli ${ }^{1}$, M. Bolzonella $^{1}$, A. Bongiorno ${ }^{10}$, \\ K. Caputi ${ }^{5}$, G. Coppa ${ }^{3,1}$, O. Cucciati ${ }^{11}$, S. de la Torre ${ }^{7}$, L. de Ravel ${ }^{7}$, P. Franzetti $^{2}$, B. Garilli ${ }^{2}$, A. Iovino ${ }^{11}$, \\ P. Kampczyk ${ }^{5}$, J.-P. Kneib ${ }^{7}$, C. Knobel ${ }^{5}$, K. Kovač ${ }^{5}$, F. Lamareille ${ }^{6,1}$, J.-F. Le Borgne ${ }^{6}$, V. Le Brun ${ }^{7}$, C. Maier ${ }^{5}$, \\ R. Pellò ${ }^{6}$, Y. Peng ${ }^{5}$, E. Perez Montero ${ }^{6}$, E. Ricciardelli ${ }^{9}$, C. Scarlata ${ }^{5}$, J. D. Silverman ${ }^{5,8}$, M. Tanaka ${ }^{8}$, L. Tasca $^{7}$, \\ L. Tresse ${ }^{7}$, E. Zucca ${ }^{1}$, U. Abbas ${ }^{7}$, D. Bottini ${ }^{2}$, P. Capak ${ }^{12}$, A. Cappi ${ }^{1}$, P. Cassata ${ }^{7}$, M. Fumana ${ }^{2}$, L. Guzzo ${ }^{11}$, \\ A. Leauthaud ${ }^{7}$, D. Maccagni ${ }^{2}$, C. Marinoni ${ }^{13}$, H. J. McCracken ${ }^{14}$, P. Memeo ${ }^{2}$, B. Meneux ${ }^{11}$, P. Oesch ${ }^{5}$, C. Porciani ${ }^{5}$, \\ R. Scaramella ${ }^{15}$, and N. Scoville ${ }^{12}$ \\ (Affiliations can be found after the references)
}

Received 4 July 2008 / Accepted 6 October 2008

\begin{abstract}
Aims. We investigate the relationships between three main optical galaxy observables (spectral properties, colors, and morphology), exploiting the data set provided by the COSMOS/zCOSMOS survey. The purpose of this paper is to define a simple galaxy classification cube, with a carefully selected sample of $\approx 1000$ galaxies.

Methods. Using medium resolution spectra of the first $1 \mathrm{k}$ zCOSMOS-bright sample, optical photometry from the Subaru/COSMOS observations, and morphological measurements derived from ACS imaging, we analyze the properties of the galaxy population out to $z \sim 1$. Applying three straightforward classification schemes (spectral, photometric, and morphological), we identify two main galaxy types, which appear to be linked to the bimodality of galaxy population. The three parametric classifications constitute the axes of a "classification cube".

Results. A very good agreement exists between the classification from spectral data (quiescent/star-forming galaxies) and the one based on colors (red/blue galaxies). The third parameter (morphology) is not as well correlated with the first two; in fact, a good correlation between the spectral classification and the classification based on morphological analysis (early-/late-type galaxies) is achieved only after partially complementing the morphological classification with additional color information. Finally, analyzing the 3D-distribution of all galaxies in the sample, we find that about $85 \%$ of the galaxies show a fully concordant classification, being either quiescent, red, bulge-dominated galaxies ( 20\%) or star-forming, blue, disk-dominated galaxies $(\sim 65 \%)$. These results imply that the galaxy bimodality is a consistent behavior both in morphology, color, and dominant stellar population, at least out to $z \sim 1$.
\end{abstract}

Key words. galaxies: general - galaxies: evolution - galaxies: fundamental parameters

\section{Introduction}

Galaxies have a wide variety of physical and observational properties. However, since the early works of Humason (1936) and Hubble (1938), it has been known that the morphologies of galaxies correlate with their colors and consequently with the derived properties of their stellar population. Big efforts have been made to establish reliable relationships between

* Based on observations undertaken at the European Southern Observatory (ESO) Very Large Telescope (VLT) under Large Program 175.A-0839. Also based on observations with the NASA/ESA Hubble Space Telescope, obtained at the Space Telescope Science Institute, operated by AURA Inc., under NASA contract NAS 5-26555, with the Subaru Telescope, operated by the National Astronomical Observatory of Japan, with the telescopes of the National Optical Astronomy Observatory, operated by the Association of Universities for Research in Astronomy, Inc. (AURA) under cooperative agreement with the National Science Foundation, and with the Canada-France-Hawaii Telescope, operated by the National Research Council of Canada, the Centre National de la Recherche Scientifique de France, and the University of Hawaii.

$\star \star$ Present address: Department of Physics and Astronomy, University of Glasgow, Glasgow G12 8QQ, UK. the morphological types, colors, and spectral characteristics of the galaxies (i.e. Fioc \& Rocca-Volmerange 1999; Bershady et al. 2000). Accurately determining these relationships is of paramount importance to our understanding of galaxy formation and evolution. The main galaxy optical properties are the spectral energy distributions, spectral line properties, internal velocity dispersions, morphologies, sizes, and structural components. From these observable quantities, we can derive intrinsic physical properties, such as stellar and gaseous masses, metallicities, and star-formation histories; each of these aspects provides crucial clues as to how galaxies were created and have evolved. The most basic evolutionary processes and their corresponding observables are, however, not yet clear and we are still uncertain whether the correlations between galaxy properties observed are driven by fundamental aspects of the galaxy population (Conselice 2006). The goal of this paper is to investigate the relationships between the three main optical galaxy observables (spectral properties, colors, and morphology) by taking advantage of the unique data set provided by the COSMOS/zCOSMOS survey.

A fundamental property discovered by large surveys, such as the Sloan Digital Sky Survey (SDSS) and the Two-Degree 
Field Galaxy Redshift Survey (2dFGRS), is that the optical color distribution of galaxies at low redshift can be represented by a bimodal function (Strateva et al. 2001; Wild et al. 2005). The bimodality separates the locus of star-forming galaxies from that of early-type galaxies (ellipticals, $S 0$, and early-type spirals), such that the fraction of the red, passive population increases progressively with the luminosity, becoming less than the blue fraction for $M_{r}>-20$ and dominating the distribution for $M_{r}<-21$ (Baldry et al. 2004). The bimodality is also present in different aspects of the galaxy population: structural parameters such as concentration index (Ball et al. 2006) and disk-to-bulge ratio (Drory \& Fisher 2007), and spectral features such as D4000 and line ratios (Kauffmann et al. 2003; Balogh et al. 2004a; Yan et al. 2006; Franzetti et al. 2007), all exhibit strong bimodal distributions. The bimodality in galaxy properties, clearly evident in the local Universe, is still present at $z \sim 1$ (Bell et al. 2004), but it is less clear whether the two distinct galaxy populations hold at higher redshifts (up to $z=2$; Giallongo et al. 2005; Cirasuolo et al. 2007; Cassata et al. 2008). A key test for galaxy evolution models is to explain why this bimodality occurs. It is still unclear which processes produce two sets of galaxies of different average colors and dispersions and different luminosity ranges.

Determining the nature of the bimodality can be approached by means of large spectroscopic redshift surveys, studying the measured galaxy parameters as a function of redshift, luminosity, and spectral properties. The zCOSMOS survey opens the possibility to extend the study of bimodality out to $z \sim 1$.

zCOSMOS is a large redshift survey that is being undertaken in the COSMOS field as an ESO Large Program $(\sim 600 \mathrm{~h}$ of observation) with the VIMOS spectrograph at the VLT. The survey consists of two parts: the first part targets a magnitudelimited sample selected from ACS images to have $I_{\mathrm{AB}}<22.5$ and observed at medium resolution. This will provide spectra and redshifts for $\approx 20000$ galaxies out to $z \sim 1.5$ over two square degrees. The second and deeper part of the survey is acquiring spectroscopy to measure the redshifts of $\approx 12000$ galaxies at $1.4<z<2.5$ at lower resolution and over the central square degree, by selecting targets using various color-selection criteria.

This paper, based mainly on the spectroscopic data of the zCOSMOS survey, takes advantage of the large potential of the COSMOS database. We present the analysis of a representative sample of the bright component of the full survey, consisting of around 1200 galaxies observed during the first year of observations. Using the medium resolution spectra of these objects and complementing them with the optical photometry from the COSMOS multi-band catalog (Capak et al. 2007) and morphological information derived from ACS imaging (Scoville et al. 2007; Scarlata et al. 2007a), we investigate the optical properties of the galaxy population out to $z \sim 1$. Applying three straightforward classifications (spectral, photometric, and morphological), we are able to distinguish two main galaxy types, which are intimately connected with the bimodality and formation history of the galaxy population: the three parametric classifications constitute the axes of a "classification cube". It is important to notice that we develop our cube using only observational parameters, such as emission-line equivalent widths, continuum indices, photometric colors (and not synthetic colors), and a nonparametric morphological classification. The choice is motivated by the desire to avoid dependence on models, galaxy templates, and parametrization of galaxy properties. Moreover, using simple observed properties makes the classification cube easy to replicate in other galaxy surveys.
The principal aim of this paper is to define the galaxy classification cube using a fair but relatively small sample of $\approx 1000$ galaxies. The full exploitation of the classification cube, applied to larger galaxy samples (i.e. the $10 \mathrm{~K}$ zCOSMOS sample, Lilly et al. 2008, in preparation), will be presented in a forthcoming paper (Coppa et al., in preparation).

\section{Description of zCOSMOS observations and data reduction}

For an exhaustive discussion of the zCOSMOS survey design, in particular of the assembly of the input catalogs, the target selection, and the spectroscopic mask design, we refer the reader to the survey presentation paper (Lilly et al. 2007).

The brighter part of zCOSMOS project consists of spectroscopic data of targets identified by means of a pure magnitudelimited selection $I_{\mathrm{AB}}<22.5$, as used in the CFRS (Lilly et al. 1995) and VVDS-wide surveys (Le Fèvre et al. 2005). This selection culls galaxies mainly in the redshift range $0.1<z<1.2$. A medium resolution grism $(R \sim 600)$ has been used with a slit width of 1 arcsec, to achieve a velocity accuracy of $\sim 100 \mathrm{~km} \mathrm{~s}^{-1}$, and with one hour integrations to enable redshifts to be measured with a high success rate. The spectral range of observations is typically 5550-9650 $\AA$, which allows the measurements of strong diagnostic lines, centered on a rest-frame wavelength of approximately $4000 \AA$, out to a redshift of about 1 . The onehour total integration is acquired in five $720 \mathrm{~s}$ exposures, between which the telescope was placed at different offset positions to complete an optimal sky subtraction, in a pattern of positions separated by one arcsec along the slit. The observations were executed during periods when the seeing was better than 1.2 arcsec. The first zCOSMOS-bright spectroscopic observations were completed in VLT Service Mode between April and June 2005. Eight sets of VIMOS mask observations were completed, which provided spectra of 1303 objects, corresponding to about $6 \%$ of the planned bright survey program. The data reduction was carried out using the VIPGI software, developed specifically for data acquired by VIMOS instrument (Scodeggio et al. 2005). Lilly et al. (2007) described the redshift determination procedures and the assignment of confidence classes to each measured redshift. The confidence in the redshift determination was indicated by a flag, whose values were: 4 (completely secure redshift); 3 (very secure redshift, but with a very marginal possibility of error); 2 (a likely redshift, but with a significant possibility of error); 1 (possible redshift); 0 (no redshift determination); and 9 (redshift based on a single strong emission line). The high quality of the photometric measurements for the COSMOS field and the corresponding photometric redshifts at $z<1.2$ (Feldmann et al. 2006), implied that the photometric redshifts could be used to assess the spectroscopic redshift reliability of our zCOSMOS-bright spectra. To indicate the reliability of a redshift, we added a decimal place to its confidence flag: if the difference between the photometric and spectroscopic redshift, $\left|z_{\mathrm{p}}-z_{\mathrm{s}}\right|$, was less than $0.1 \times\left(1+z_{\mathrm{s}}\right)$, we added the decimal place $=.5$; otherwise, we added a decimal place $=.1$. In the following, we include in the spectroscopic "high quality" data set all the galaxy spectra with flags 3 and 4, and those with flag 2.5, which includes galaxy spectra with flag $=2$ confidence for which the photometric and spectroscopic redshifts are in good agreement. We exclude from the high-quality data set galaxy spectra of flag 9, where the redshift could be determined using only one emission line. Although this flag corresponds to significant detection of an emission line, the absence of other spectroscopic 

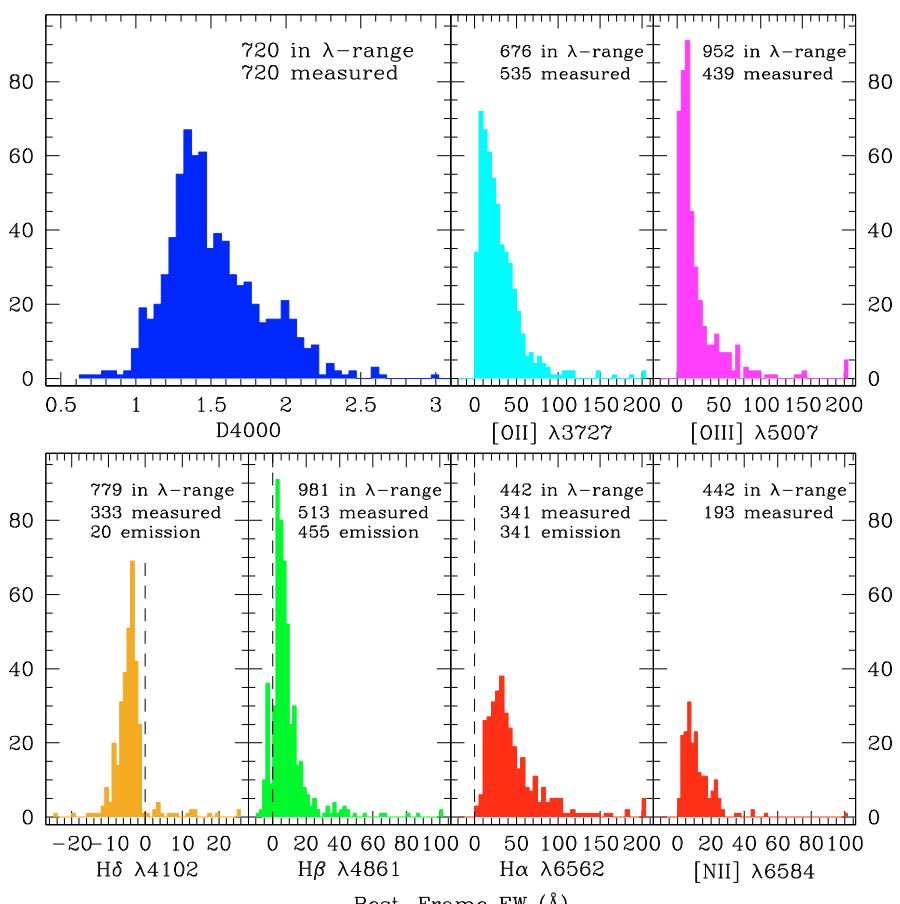

Fig. 1. Rest-frame measurement distributions of the analyzed spectral features, from top-left to bottom-right: D4000, and $E W$ of the lines [OII] $\lambda 3727,[\mathrm{OIII}] \lambda 5007, \mathrm{H} \delta, \mathrm{H} \beta, \mathrm{H} \alpha$, and [NII] $\lambda 6584$. In each panel, we indicate the number of galaxies for which the feature is within the spectral observed range and the number of measured lines.

diagnostic lines implies that, for many, the continuum signal-tonoise ratio (hereafter $S / N$ ) could be rather low.

\section{Spectral analysis}

\subsection{Spectral measurements}

We performed spectral measurements for a total of 1130 galaxies (and 20 QSOs) observed during the first year of observations and for which a redshift had been determined. This set of extragalactic objects forms the analyzed $1 k$ zCOSMOS-bright sample.

We measured the spectral quantities using semi-automatic procedures that were adaptations of the IRAF task splot. First, the continuum was fitted automatically to fixed wavelength intervals, although our procedures also enabled interactive adjustments of the continuum level to improve the line measurement in noisy spectra. Then, equivalent widths $(E W s)$ and fluxes were measured by applying both a Gaussian-function fitting algorithm and a direct integration of the continuum-subtracted line profiles. The fluxes measured by both techniques were in excellent agreement: for all emission lines, the two measurements agreed to within $25 \%$, while the $1-\sigma$ dispersion of the difference distribution corresponded to only $11 \%$. Figure 1 shows the distributions for principal spectroscopic line measurements for the $1 k$-sample. The equivalent widths are given in the rest-frame and indicated by positive or negative values for emission or absorption lines, respectively. Errors in the measured equivalent widths were estimated by taking into account both the measured rms of the continuum close to the lines and the difference between the values derived by the two techniques. The median uncertainty in an individual $E W$ is $0.7 \AA$, but it is worth noting that we did not account for the stellar absorption in the measurements of the Balmer emission lines, since in most cases the $S / N$ of

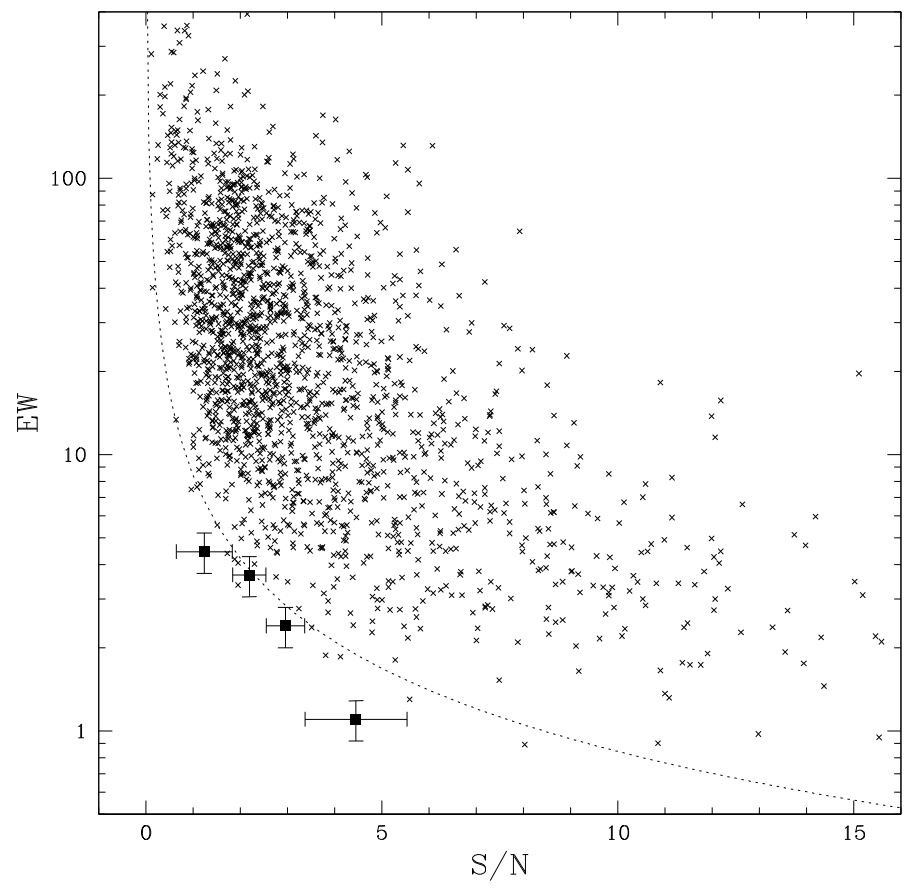

Fig. 2. Observed $E W \mathrm{~s}$ of all the measured emission lines (see Fig. 1) versus the $S / N$ of the continuum close to the line; the lower envelope of the measured equivalent widths is fitted well by the dotted curve (see Eq. (1) in the text). The larger filled square symbols represent the [OII] $E W$ values measured in composite spectra of galaxies that individually did not demonstrate a detectable emission line, grouped in four equally populated bins according to their continuum $S / N$; the line $E W \mathrm{~s}$ have been measured in the composite spectra shifted to mean redshift of the corresponding galaxy group. The horizontal error bars indicate the range of $S / N$ included in each bin, whereas the vertical error bars show the variation in the observed $E W$ if the composite spectra were moved to the extremes of the redshift range.

our spectra did not enable a simultaneous fit of both the absorption and emission components. We measured $E W$ s and fluxes for [OII] $\lambda 3727, \mathrm{H} \delta, \mathrm{H} \beta,[\mathrm{OIII}] \lambda 5007, \mathrm{H} \alpha$, and [NII] $\lambda 6584$, as well as the strength of the $4000 \AA$ break (D4000; Bruzual 1983). The continuum fluxes were measured in fixed wavelength ranges, and our procedure computed the average using a sigmaclipping technique, to ensure that spikes due to bad sky subtraction and/or to cosmic-ray residuals did not affect the measured values significantly. Errors in the continuum fluxes were then computed from the standard deviation. We focused our analysis on $E W$ measurements and flux ratios since the absolute spectrophotometry was not completely reliable because of the corrections for slit losses that had been applied to the spectra. This set of "semi-automatic" measurements was used, in addition, to test and calibrate the fully automatic measurement code PlateFit (Lamareille et al. 2006), which is being applied to larger zCOSMOS data sets.

To estimate our emission line detection limits, we measured the $S / N$ in the continuum adjacent to the lines. Figure 2 shows the relation between the $S / N$ in the continuum and the observed equivalent widths of the measured emission lines in our sample: from this figure, it is clear that a correlation exists between the minimum detected $E W$ and the continuum $S / N$. The lower envelope of the measured $E W$ s (dotted line in Fig. 2) is described well by the curve

$E W($ detection limit $)=\frac{S L * \Delta}{(S / N)_{\text {cont }}}$, 


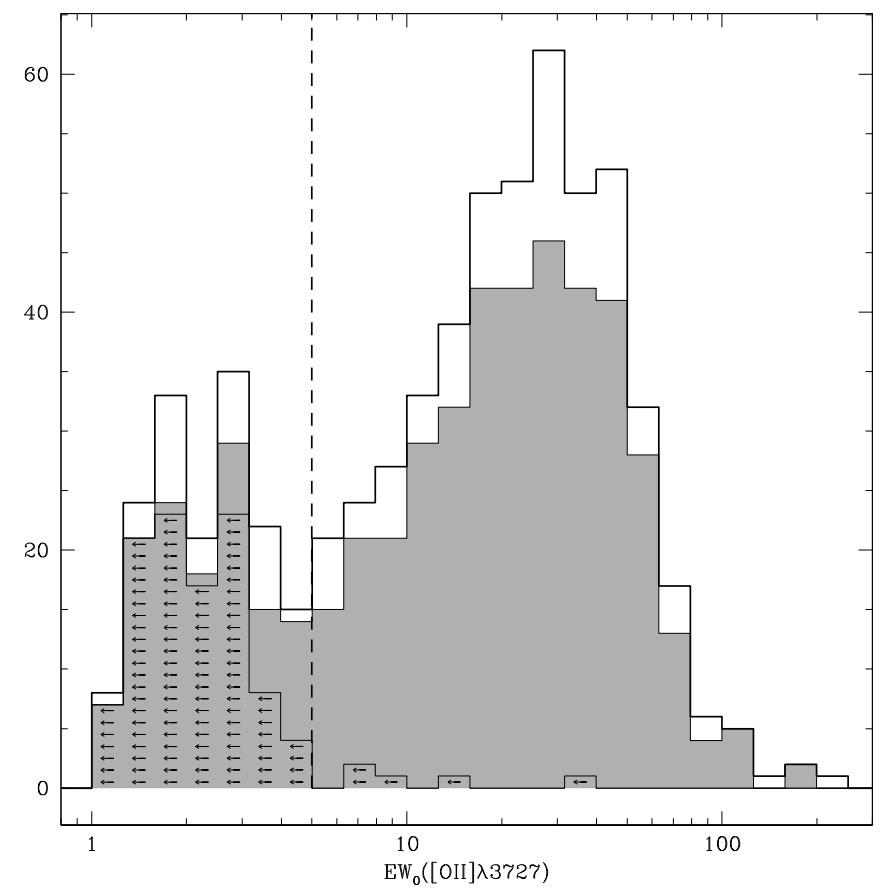

Fig. 3. Distribution of the rest-frame [OII] $E W s$ for the zCOSMOS galaxy $1 k$-sample. The gray histogram represents the parameter distribution for galaxies included in the high-quality data set, with leftward arrows indicating the upper limits calculated using Eq. (1); the thin empty histogram is for the whole sample.

where $\Delta$ is the resolution element (in $\AA$ ) of our spectra and $S L$ is the significance level of the detectable line, expressed in terms of sigma of the continuum noise (Colless et al. 1990; Manning 2002). On the basis of Fig. 2, we adopt an $S L(\sigma)=3.5$. Measuring the $S / N$ in the continuum of each galaxy spectra without a detectable emission line, we estimate the $E W$ upper limits (using Eq. (1)). The robustness of this technique in estimating the line upper limits is demonstrated by the detection of the emission lines (in particular [OII] 13727) in composite spectra of galaxies without a detectable emission line. We grouped 141 single spectra in four equally populated bins according to their continuum $S / N$, and all the measured [OII] $E W$ s lie below the curve calculated using the above equation (see Fig. 2).

\subsection{Galaxy spectral classification}

Following Mignoli et al. (2005), we applied to the zCOSMOS-bright galaxy data set the straightforward spectral classification scheme adopted for the $K 20$ survey (Cimatti et al. 2002), which uses the equivalent width of the [OII] $\lambda 3727$ line and the $4000 \AA$ break index to define two main spectral classes: red quiescent galaxies, which show a high $D 4000$ value and a faint, if not undetected, [OII] emission line; and blue star-forming galaxies, with low D4000 and intense emission lines. This spectral classification scheme naturally arises from the intrinsic bimodal nature of galaxy physical properties that has been observed in the color distribution (Balogh et al. 2004b; Bell et al. 2004) and mean spectroscopic indices (Kauffmann et al. 2003; Franzetti et al. 2007). In Fig. 3, the distribution of the rest-frame [OII] equivalent widths is shown for the zCOSMOS galaxy $1 k$-sample. A clear bimodality is evident in the $E W_{0}([\mathrm{OII}])$ distribution, especially if we consider that the histogram with leftward arrows represents the upper limits to line measurements. On the basis of this behavior, we separate the galaxy population into two classes, strong and weak line emitters, divided by an $E W_{0}([\mathrm{OII}])=5 \AA$. With this threshold, almost all the objects with undetected emission lines are included in the weak emission-line galaxy group. Most galaxies with high $E W$ that are not included in the high-quality sample are flag $=9$ objects (see end of Sect. 2).

Looking at Fig. 4, a couple of comments can be made. First, the "low-density" region in the leftward corner of the classification plane is populated mainly by poor-quality flagged objects (indicated by crosses in the figure), confirming both the doubtful nature of their redshift identification and the efficiency of the spectral classification scheme. The galaxies in this region of the plane are not spectroscopically classified. Furthermore, when applied to the high-ranked zCOSMOS galaxies, the classification scheme demonstrates its efficiency: only four objects fall marginally in the leftward region of the plot, whereas the intermediate class (red emission-line galaxies) constitutes only $4 \%$ of the sample. Interestingly, the few (broad line) active nuclei observed in the useful redshift range are quite segregated in the classification plane, having very blue continuum but relatively faint [OII] emission lines ( $<10 \AA$, Forster et al. 2001).

Figure 4 shows the resulting spectral classification plane. Due to the high quality of zCOSMOS spectroscopic data, higher than in the $K 20$ survey, we are able to appreciate how most of the galaxies with intense emission lines occupy a narrow region in the D4000-[OII] plane. We performed an iterative linear least squares fit with $\sigma$-clipping, to define and delimit the locus of these objects (see diagonal dashed lines in Fig. 4). The final criterion for defining the blue emission-line galaxies is

$$
\begin{aligned}
& E W_{0}([\mathrm{OII}]) \geq 5 \AA \\
& 1.50 \leq D 4000+0.33 \times \log \left(E W_{0}([\mathrm{OII}])\right) \leq 2.22
\end{aligned}
$$

A relationship between the $D 4000$ and the [OII] equivalent width was expected, since both features are related to the galaxy star-formation history, either the most recent one $\left(E W_{0}([\mathrm{OII}])\right)$ or its integral over time (D4000). It is particularly encouraging that, by projecting our linear fit in the $D 4000$-specific SFR plane, with the simple assumption of constant mass-to-luminosity ratio, we almost exactly reproduce the mode of the SDSS data distribution presented in Fig. 11 of Brinchmann et al. (2004).

It is worth keeping in mind that the observed spectral range enables us to apply this spectral classification scheme only to galaxies of sufficiently high redshift to include the [OII]$D 4000$ region, i.e. the redshift range $z \sim 0.45-1.25$. Within this redshift range, 631 galaxies were classified, of which 511 belong to the high-quality sample on the basis of the assigned redshift flag. More than $80 \%$ of the spectroscopically classified zCOSMOS galaxies belong to the two previously defined classes: red quiescent galaxies and blue star-forming galaxies. A third group of objects, which have emission lines but a red stellar continuum (as implied by their high value of $D 4000$ ), constitutes what we refer to as the intermediate class of galaxies. Interestingly, 20\% of these objects show some sign of nuclear activity: three of the twenty galaxies in this class are classified as Seyfert 2 galaxies (Bongiorno et al., in preparation) on the basis of the diagnostic diagrams of Baldwin et al. (1981), and one additional galaxy has an X-ray counterpart (Brusa et al. 2007). In contrast, the fraction of the blue star-forming galaxy population that are candidate AGN is only $2 \%$, i.e. 7 out of 361 galaxies.

Composite spectra for each galaxy class were generated by averaging all spectra included in that class. To create the composites, each spectrum was shifted to the rest-frame 

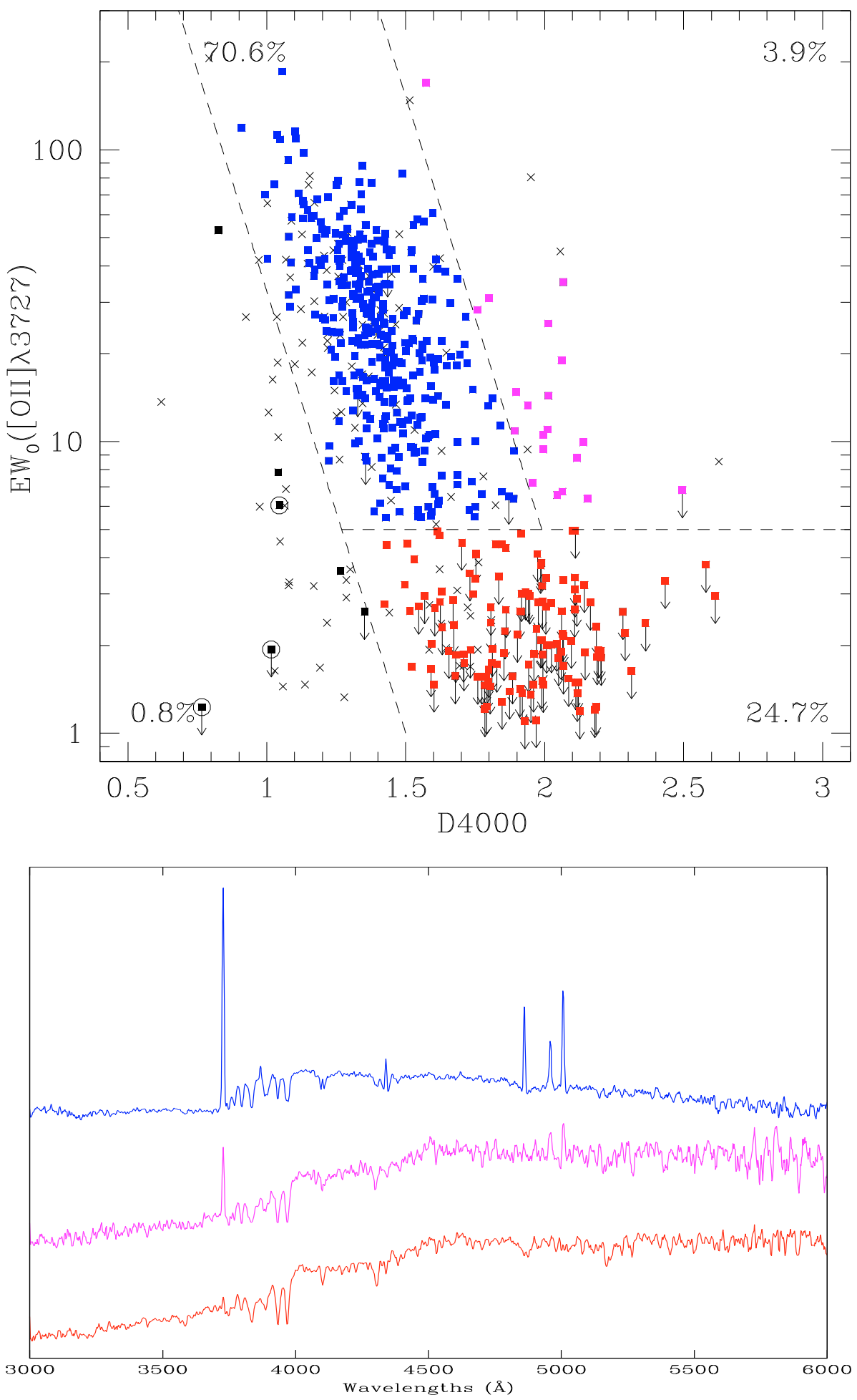

Fig. 4. The classification plane for the $1 k$ zCOSMOS galaxies; using the rest-frame [OII] $\lambda 3727 \mathrm{EW}$ and D4000, we define three main classes: red quiescent galaxies (lower right region), blue emission-line galaxies (upper left region), and the intermediate galaxies, which show emission lines but a high value of $D 4000$. In the figure, we plot all classifiable galaxies (with $z \sim 0.45 \div 1.25$ ), with different symbols following the redshift quality ranks (crosses for the low-quality spectra, filled squares for the high-quality ones). Broad line objects (quasars) are encircled in the figure. The dashed lines represent the boundaries of the adopted classification scheme following Eq. (2). The percentage of high-quality galaxies falling in each classification regions are labelled at plot corners.
Fig. 5. Composite spectra of the three main galaxy classes as defined in Fig. 4. From bottom to top: 126 red quiescent, 20 intermediate, and 361 blue star-forming galaxies contribute to the averages. according to its redshift (with a $1.5 \AA$ rest-frame bin ${ }^{1}$ ) and normalized in the 4000-4500 $\AA$ wavelength range, which was always present in the observed spectroscopic window. An identical weight was assigned to each individual spectrum, to avoid biasing the final composite towards the brightest galaxies. Finally, all spectra belonging to a particular spectroscopic class were stacked. In Fig. 5, the average spectra of the three main

\footnotetext{
${ }^{1}$ At the median redshift of the analyzed galaxy sample $(z=0.724)$, a rest-frame bin of $1.5 \AA$ matches the pixel size used in the spectroscopic observations.
}

spectroscopic classes are plotted. The continuum in the composite of the emission-line galaxies with red D4000 is largely indistinguishable from that of the purely passive galaxies composite. Therefore, this class of intermediate objects should consist mainly of galaxies undergoing a modest star-formation episode, rather than of heavily reddened star-forming galaxies. The presence of the [OIII] emission doublet, with no evidence of $\mathrm{H} \beta$ in emission, in the composite of intermediate objects could indicate some nuclear activity (i.e. type II AGNs) contamination in this galaxy class, as already noted above (see also Yan et al. 2006). 


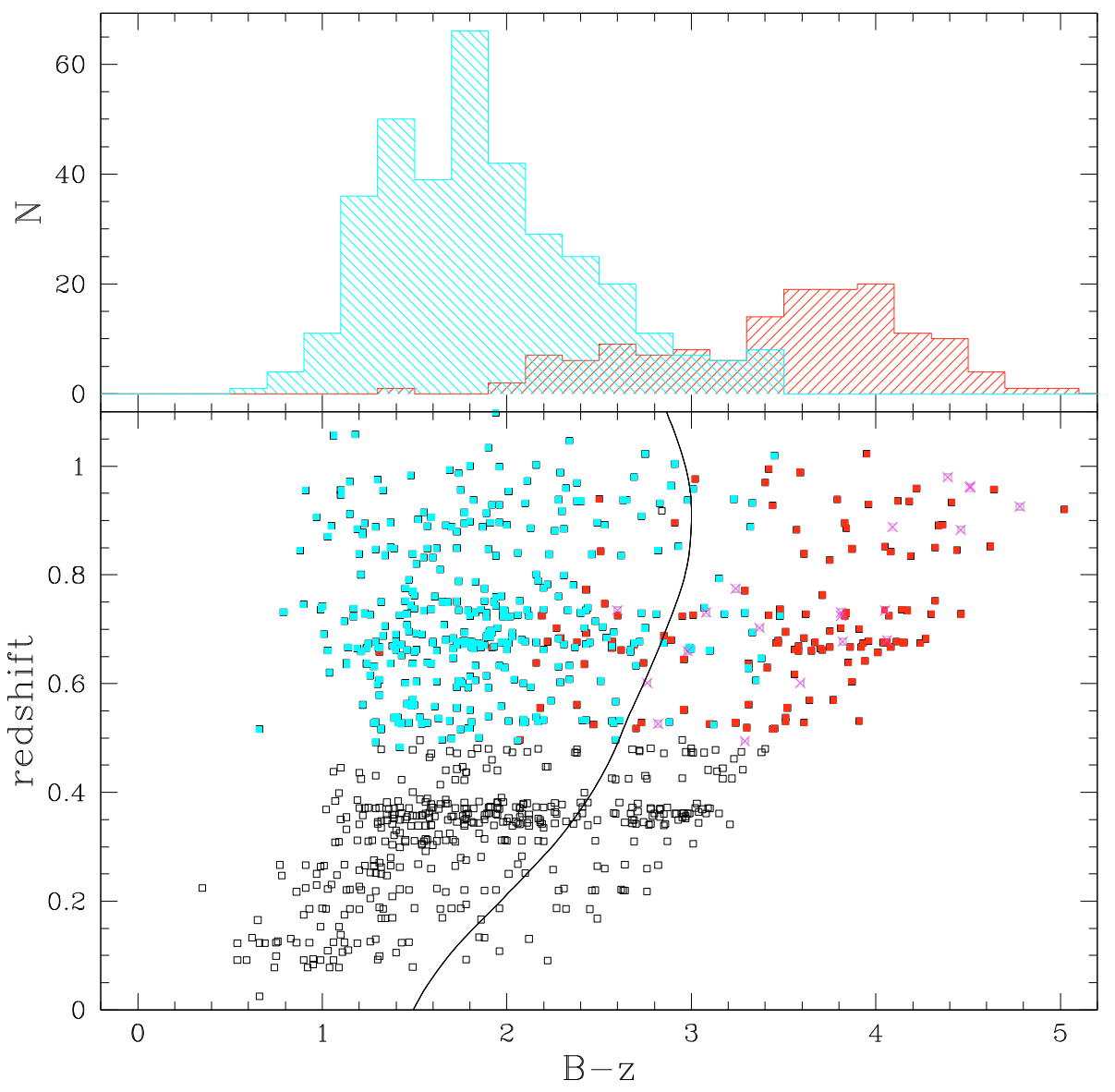

Fig. 6. Upper panel: observed $B-z$ color distribution of the galaxies belonging to zCOSMOSbright $1 k$-sample with $0.45 \leq z \leq 1.2$; cyan histogram for blue star-forming, red histogram for quiescent galaxies, both with and without emission lines. Lower panel: $B-z$ versus redshift plot for the whole high-quality sample; cyan and red squares for star-forming and quiescent galaxies respectively, magenta crosses for intermediate ones). We divide the two galaxy populations using as discriminator the $B-z$ track of the Sab template from the Coleman et al. Extended set (solid line in figure; Sawicki et al. 1997).

\section{Photometric colors of galaxy spectral classes}

The bimodality in the galaxy spectroscopic index measurements identified above may be naturally linked to the color bimodality observed widely in the galaxy population. We therefore compare the observed colors and spectral classification of galaxies that belong to the zCOSMOS-bright $1 k$-sample.

The photometric observations of the COSMOS field that we use in our study include optical $\left(B_{J}, g^{+}, V_{J}, r^{+}, i^{+}\right.$, and $z^{+}$data acquired using SuprimeCam at the Subaru Telescope, $u^{*}$-band data with the Canada-France-Hawaii Telescope and $i_{814}$ images with $\mathrm{ACS} / \mathrm{HST})$ and near-Infrared $\left(K_{\mathrm{s}}\right.$-band data acquired using the Cerro Tololo International Observatory and Kitt Peak National Observatory telescopes) wavelengths. Details of the ground-based observations and data reduction are presented in Capak et al. (2007) and Taniguchi et al. (2007). The reduced images in all bands were PSF-matched by Gaussian convolution with a final FWHM corresponding to the seeing in the $K_{\mathrm{S}}$ band (1.5"); the multi-band photometry catalog was then generated using SExtractor (Bertin \& Arnouts 1996). First, the total (SExtractor magauto) and fixed-aperture ( $3^{\prime \prime}$-diameter) magnitudes were measured on the detection image ( $i$-band), allowing the estimate of the aperture correction for each galaxy. This correction was subsequently applied to aperture magnitudes measured in the other optical/near-IR bands. Details of the photometry, star/galaxy separation, and catalog construction were provided by Capak et al. (2007).

We analyzed different colors from the photometric catalog and attempted to identify a separation in photometric space between the spectral galaxy classes defined above. The $B-z$ color, in addition to both $B-K$ and $u-i$, appears to provide the most effective way of separating red quiescent and blue star-forming galaxies. In the upper panel of Fig. 6, we illustrate, with the $B-z$ distribution histograms, the good separation between the color distribution of the different spectral classes, although with some overlap for $2<B-z<3$. We verified whether the slit aperture effects would bias the comparison between the observed colors and spectral classification: the slit corrections, estimated on the basis of the difference between the integrated magnitudes computed from the flux-calibrated spectra and the $I$-band photometric data, do not show any trend with either the magnitude or $B-z$ color in the analyzed sample.

The correlation between color and spectral classification becomes even tighter when we consider galaxy redshifts. The $B-z$ versus redshift plot (lower panel of Fig. 6) exhibits a clear segregation between the galaxy classes. To divide the two galaxy populations effectively, we adopted the $B-z$ versus redshift evolutionary track of a template spectrum from the Coleman et al. Extended set (Coleman et al. 1980; Sawicki et al. 1997) with properties corresponding most closely to those dividing the two populations. The chosen template had the optical colors (Fukugita et al. 1995) and spectral appearance (Kennicutt 1992) of an Sab galaxy. Also in local galaxy samples (i.e. The Revised Shapley-Ames Catalog of Galaxies; van den Bergh 2007), the transition between blue and red galaxies occurs at the Hubble type $\mathrm{Sab} / \mathrm{Sb}$.

The $B-z$ versus redshift plot shows that almost all of the small number of discordant galaxies (blue quiescent and red starforming) fall close to the separation track in the color classification plane, confirming the agreement between the spectral and photometric classifications. Moreover, galaxies of the intermediate spectral class (red D4000 with emission lines, magenta crosses in Fig. 6) have a color distribution similar to that of quiescent galaxies, in agreement with the observed similarity 
Table 1. Numbers of galaxies in the spectroscopic and photometric classes.

\begin{tabular}{cccc}
\hline \hline & \multicolumn{3}{c}{ Spectral classification } \\
$B-z$ & Quiescent & Star-forming & Total \\
\cline { 2 - 4 } Red & 119 & 20 & 139 \\
Blue & 27 & 335 & 362 \\
TOTAL & 146 & 355 & 501 \\
\hline
\end{tabular}

of the stellar continuum in the composite spectra noted above (Fig. 5).

By merging these two spectral classes and using the track of the Sab-template to separate the photometrically "blue" and "red" galaxies, we compiled a $2 \times 2$ contingency table for the high-quality sample (Table 1), useful in performing a statistical analysis of the relationship between the spectral and photometric classifications. To quantify the degree of correlation between the two classification schemes, we computed Pearson's linear correlation coefficient for the data presented in Table 1: the two classifications are well-correlated, as indicated by a Pearson coefficient of 0.77 .

In the following, we summarize how the different galaxy populations inhabit the color versus spectral classification plane:

- $82 \%$ of objects classified as "quiescent" from spectra are red;

- $94 \%$ of objects classified as "star-forming" from spectra are blue;

- $86 \%$ of objects with red colors are "quiescent" from the spectra;

- $93 \%$ of objects with blue colors are "star-forming" from the spectra.

- The fraction of outliers is $\sim 9 \%(47 / 501)$.

The correspondence between the two classifications is not optimal for the $18 \%$ quiescent galaxies that show bluish colors. We investigated the nature of these objects by studying their composite spectrum. The continuum in the composite of blue quiescent galaxies is largely indistinguishable from that of the red, quiescent galaxies, but the spectrum shows a moderate [OII] emission line $\left(E W_{0}=4.4 \AA\right)$ and a strong $\mathrm{H} \delta$ in absorption $\left(E W_{0}=-3.8 \AA\right)$. The presence of these two spectral features represents star formation activity, which could also explain the bluish colors of this class of galaxy. The restframe equivalent width of [OII], measured in the composite spectrum, is below the cut adopted for the spectral classification $\left(E W_{0}([O I I])>5 \AA\right)$, and only one of the 27 blue quiescent galaxies is spectroscopically classified as intermediate. Therefore, it is more reasonable to consider these objects as being galaxies in-between the two main spectral classes, rather than galaxies of extremely discordant spectral and color properties. Indeed, about $30 \%$ of these galaxies can be spectroscopically classified as post-starburst galaxies and constitute the $64 \%$ of the $1 k$ sample of post-starburst galaxies (7/11; Vergani et al., in preparation).

The good agreement between the classification from spectral data (quiescent/star-forming galaxies) and that based on colors (red/blue galaxies) is achieved within the relatively bright $\left(I_{\mathrm{AB}}<22.5\right)$ zCOSMOS sample; using the deeper $\left(I_{\mathrm{AB}}<24.0\right)$ VVDS survey, Franzetti et al. (2007) have demonstrated that the incidence of red star-forming or blue quiescent galaxies appear to increase when studying fainter galaxy samples.

\section{ACS morphology of galaxy spectral classes}

The HST/ACS imaging is an integral component of the COSMOS survey, providing very high sensitivity and high resolution imaging over a large field of view. These space-based images yield resolved morphologies for several hundreds of thousands galaxies, and the zCOSMOS-bright survey magnitude limit $\left(I_{\mathrm{AB}}<22.5\right)$ is matched with the $I$-band ACS depth to allow classical bulge-disk decomposition. The COSMOS survey is a natural "battlefield" for competing morphological classification schemes. Among all the possible and equally good techniques implemented by the various teams within the COSMOS collaboration, we selected as our morphological classification tool the Zurich Estimator of Structural Types (ZEST; Scarlata et al. 2007a). We adopted this approach mainly because ZEST classifies galaxy types by applying a variety of non-parametric techniques that measure physical parameters directly on the ACS images in a way similar to how we classify galaxies in terms of both spectroscopic and optical color data.

In brief, ZEST quantitatively describes the galaxy structure using three variables (PC1; PC2; PC3), obtained by performing a principal component analysis (PCA) in the five-dimensional parameter space defined by asymmetry $(\mathrm{A})$, concentration $(\mathrm{C})$, Gini coefficient $(\mathrm{G})$, the 2nd-order moment of the brightest $20 \%$ of galaxy pixels (M20), and the ellipticity of the light distribution $(\epsilon)$. The morphological classification is performed in the PC1-PC2-PC3 space by linking a (dominant) morphological class to different regions in the PC-space. Finally, ZEST assigns each analyzed object to a straightforward morphological type ( $=1$ for early-type galaxies, $=2$ for disk galaxies and $=3$ for irregular galaxies). Furthermore, a "bulgeness" parameter (from 0 for bulge-dominated galaxies to 3 for bulge-less disks) is also assigned to each galaxy classified as type 2 (Scarlata et al. 2007a), following the median value of the Sersic indices distribution of galaxies clustered in the PC-space. The Sersic indices were computed by Sargent et al. (2007).

We compare the spectral classification with the morphological characterization assigned by ZEST in Fig. 7, where the histograms represent the frequency distributions of the morphological types for the three main spectroscopic classes. This figure illustrates a fairly good correlation between the spectral and the morphological classification, represented by a gradual increase in the fraction of star-forming galaxies towards morphological late-types, and, similarly, an increase in spectroscopic quiescent galaxies towards the morphological early-types.

A more instructive way of looking at the correspondence between the morphological and spectral classifications is presented in Fig. 8, which shows the distribution of the morphological types in our spectral classification plane. Following the morphological characterization, the galaxies have been divided into: (i ) "spheroids", a class including both the pure ellipticals (ZEST-type $=1)$ and the bulge-dominated galaxies $(Z E S T \text {-type }=2 \quad \text { with bulgeness parameter }=0)^{2}$; (ii) "morphologically-mixed" objects, disk (ZEST-type =2) galaxies with intermediate bulgeness parameter $(=1)$; (iii) "diskdominated" galaxies with ZEST-type $=2$ and bulgeness parameter $\geq 2$; and (iv) "irregulars", with ZEST-type $=3$. The four panels of Fig. 8 demonstrate that all but one of these morphologically defined galaxy types appear to occupy the regions expected based on their spectral classification. More than $90 \%$ of the morphological ellipticals are quiescent from the spectra, as well as a large fraction of the spheroids. Also, more

\footnotetext{
2 The inclusion of the bulge-dominated disk galaxies into the same class as pure ellipticals was already proposed by Scarlata et al. (2007b).
} 


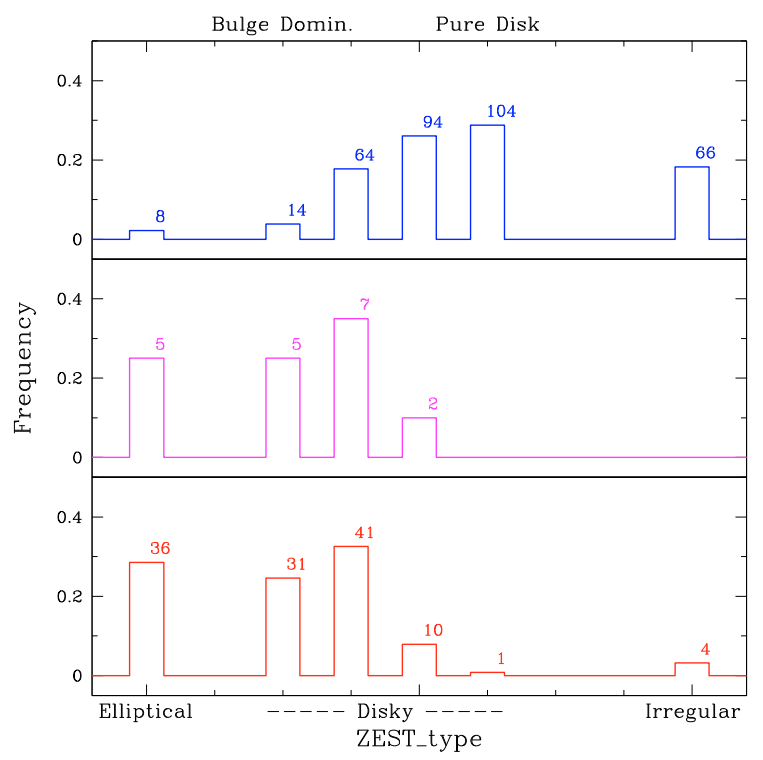

Fig. 7. Frequency distributions of ZEST morphological types for the spectroscopic blue emission-line galaxies (top histogram), the spectroscopic intermediate galaxies (i.e. galaxies which show red continuum with emission lines; middle histogram), and the quiescent galaxies (bottom histogram). The four central bins represent the most-populated ZEST class of disk galaxies, further splitted on the basis of the "bulgeness" parameter. The numbers on top of the bars indicate the number of galaxies in each bin.

than $90 \%$ of the disk-dominated and irregular galaxies have blue emission line spectra. Only the morphologically mixed objects (i.e. disk galaxies with intermediate $\mathrm{B} / \mathrm{T}$ parameters) are spread all over the spectral classification plane. Table 2 shows the distribution of ZEST types in the $1 k$-galaxy sample as a function of their spectroscopic class.

The application of a $2 \times 2$ contingency table, as a statistical test of correspondence between the spectral and morphological classifications, is not a straightforward exercise in this case, since it is difficult to assign objects with ZEST-type $=2.1$ to an early- or late- galaxy type.

Bailin \& Harris (2008) have discovered a similar population of morphologically intermediate SDSS galaxies, finding that inclination effects play an important role in their classification. Nevertheless, both a visual inspection of the ACS snapshots and a statistical analysis of the asymmetry and ellipticity parameters do not suggest the presence of an inclination bias in the zCOSMOS galaxies with ZEST-type $=2.1$. This intermediate morphological class is plausibly composed of galaxies spanning a wide range of bulge-to-disk ratios and ZEST alone is unable to identify a dominant component in their visual morphology. We decided to fully utilize the classification cube potentialities, including the photometric colors, in the analysis of this intermediate morphological class. Figure 9 shows the color distribution of ZES T-type $=2.1$ galaxies, divided following the spectral classification. The upper panel shows the distribution of the differences between the observed $(B-z)$ color and the color, at the same redshift of each galaxy, of the Sab template adopted to separate the blue and red populations in the previous section, for galaxies spectroscopically classified as star-forming; the lower panel shows the same color difference for the type $=2.1$ galaxies spectroscopically classified as quiescent. The figure clearly shows that galaxies with $Z E S T$-type $=2.1$ have $B-z$ colors that largely agree with their spectral classification: $93 \%(65 / 70)$ of the ZEST type $=2.1$ classified as "star-forming" from the

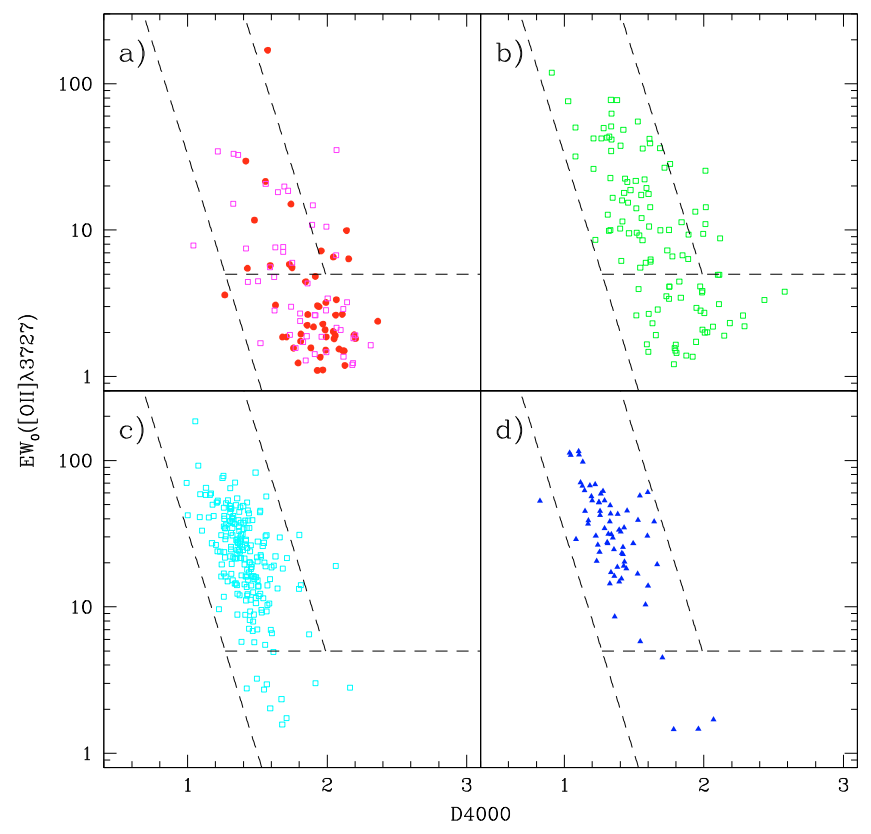

Fig. 8. Distributions of different morphological groups in our spectral classification plane. Panel a): spheroidal galaxies, including both pure ellipticals (ZEST-type $=1$; filled circles) and bulge-dominated galaxies $(Z E S T$-type $=2.0$; empty squares). Panel $\mathbf{b})$ : disk galaxies with intermediate bulgeness parameter (ZEST-type $=2.1$; empty squares $)$. Panel c): "disk-dominated" galaxies (ZEST-type $=2.2$ and $=2.3$; empty squares). Panel d): Irregular galaxies (ZEST-type $=3$; filled triangles). In all the four panels, the dashed lines indicate the spectral class boundaries.

Table 2. Numbers of galaxies in the spectroscopic and ZEST classes.

\begin{tabular}{cccc}
\hline \hline & \multicolumn{3}{c}{ Spectral classification } \\
ZES T-type & Quiescent & Star-forming & Total \\
\cline { 2 - 4 } $1.0-2.0$ & 76 & 24 & 100 \\
2.1 & 55 & 70 & 125 \\
$2.2-3.0$ & 11 & 256 & 267 \\
Total & 142 & 350 & 492 \\
\hline
\end{tabular}

spectra have blue colors, whereas $71 \%(40 / 55)$ of the ZEST type $=2.1$ classified as "quiescent" from the spectra have red colors. Interestingly, the latter galaxy class exhibits a bimodal color distribution, which is separated well by the color track of the Sab template. We investigated the average properties of the two classes of red and blue quiescent galaxies with intermediate ZEST morphology by analyzing their composite spectra. The stellar continuum appears similar in the two composites, resembling that of the normal quiescent galaxies (see Fig. 5), even if the average spectrum of the blue quiescent class has a low $S / N$ because of the small number of coadded galaxy spectra. The main difference is the presence of emission lines in the blue quiescent composite (with $E W([\mathrm{OII}]) \sim 5 \AA$ ), while emission lines are undetected in the red quiescent composite. However, the careful analysis of single galaxy subclasses is beyond the scope of this paper, and they are the subject of a forthcoming paper in which the properties of these galaxy classes will be revisited using a larger zCOSMOS data set.

Therefore, the galaxies of this morphological class have been separated by color, by assigning the red-colored type 2.1 galaxies to a spheroid class and the blue-colored type 2.1 galaxies to a disk/irregulars category. Following this recipe, a $2 \times 2$ 


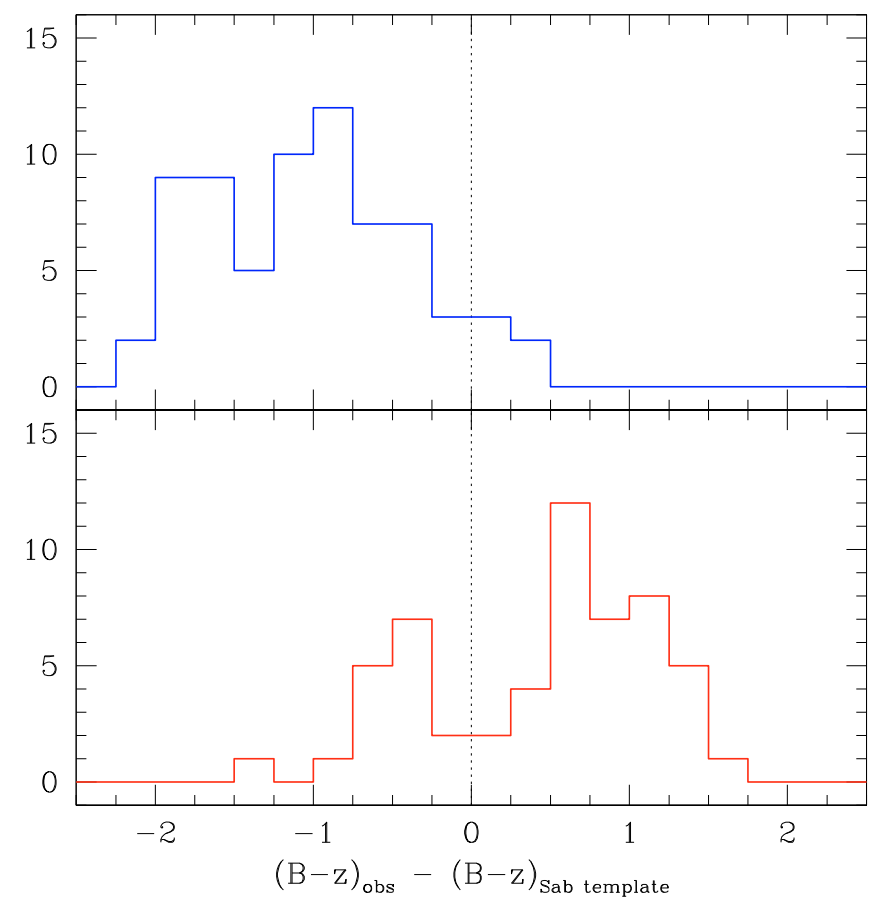

Fig. 9. The distribution of the differences between the observed $(B-Z)$ color of the type $=2.1$ galaxies and the color, at the redshift of each galaxy, of the Sab template adopted to photometrically separate the blue and red populations in Fig. 6. In the upper panel, the galaxies spectroscopically classified as star-forming; in the lower panel, the galaxies spectroscopically classified as quiescent.

Table 3. Numbers of galaxies in the spectroscopic and morphological classes.

\begin{tabular}{cccc}
\hline \hline & \multicolumn{3}{c}{ Spectral classification } \\
Morph $+(B-z)$ & Quiescent & Star-forming & Total \\
\cline { 2 - 4 } Spheroids & 115 & 29 & 144 \\
Disk/Irr & 27 & 321 & 348 \\
Total & 142 & 350 & 492 \\
\hline
\end{tabular}

contingency table has been derived (Table 3), which provide a clear indication of the correlation between the two classifications (Pearson correlation coefficient $=0.73$ ).

\section{The galaxy classification cube}

It is evident from the previous sections that strong correlations are present between the spectral classification and both the color and morphological properties of the zCOSMOS-bright galaxies. In this section, we merge these classifications into a threedimensional characterization, the galaxy classification cube. We include in our analysis all galaxies with $0.45 \leq z \leq 1.25^{3}$ and complete photometric and morphological information. The full sample includes 577 galaxies, of which 487 (84\%) belong to the high-quality spectroscopic sample on the basis of the assigned redshift flag.

A three-digit code is assigned to each galaxy of the sample, such that each digit represent one of the three classifications (spectral, photometric, and morphological, respectively). Each digit can assume a bitwise value. This corresponds to 1,

\footnotetext{
3 Since the features adopted for spectral classification (D4000 and $E W([\mathrm{OII}]))$ can be seen in the zCOSMOS-bright spectra only within this redshift range.
}

Table 4. The Classification Cube: galaxy counts and class percentages.

\begin{tabular}{crcrc}
\hline \hline $\begin{array}{c}\text { Class-code } \\
\text { s-c-m }\end{array}$ & \multicolumn{2}{c}{ High-Q. Sample } & \multicolumn{2}{c}{ All Galaxies } \\
& $\mathrm{N}$ & $(\%)$ & \multicolumn{1}{c}{$\mathrm{N}$} & $(\%)$ \\
\hline $2-2-2$ & 311 & $(63.9)$ & 374 & $(64.8)$ \\
$1-1-1$ & 105 & $(21.6)$ & 113 & $(19.6)$ \\
$1-2-2$ & 18 & $(3.7)$ & 26 & $(4.5)$ \\
$2-1-1$ & 13 & $(2.7)$ & 17 & $(2.9)$ \\
$2-1-2$ & 7 & $(1.4)$ & 8 & $(1.4)$ \\
$1-2-1$ & 9 & $(1.8)$ & 11 & $(1.9)$ \\
$2-2-1$ & 15 & $(3.1)$ & 17 & $(2.9)$ \\
$1-1-2$ & 9 & $(1.8)$ & 11 & $(1.9)$ \\
\hline Total & 487 & $(100)$ & 577 & $(100)$ \\
\hline
\end{tabular}

if the galaxy was spectroscopically classified as quiescent, had red $B-z$ color, or its morphology was dominated by the bulge component. Alternatively, we assign a digit value of 2 if the galaxy was spectroscopically star-forming, had blue $B-z$ color, or showed a disky or irregular morphology.

We recall two caveats related to both the procedure we adopt to separate galaxies into one of the two classes, and the presence of galaxies that deviate from the bimodal trends in properties (quiescent/star-forming, red/blue, bulge-dominated/diskdominated). First, a galaxy spectrum with emission lines but a red stellar continuum (implied by a relatively high value of D4000) was classified spectroscopically as quiescent because their global properties indicated that they were probably ellipticals experiencing a modest star-formation episode or including a faint/obscured AGN. Secondly, we used the $B-z$ color to determine a more robust separation of the galaxies included by ZEST in the intermediate class (ZEST type $=2.1$ ).

In Table 4, numbers and percentages of objects belonging to each cell of the classification cube are presented, using the three-digit code to identify the galaxy groups. The numbers for both the high-quality and the full sample are listed, and although all the single classification definitions were determined by using the high-quality sample, the percentages of galaxies included in each group vary only slightly between the two different samples. The main result emerging from the 3D analysis of the optical properties of the zCOSMOS-bright galaxies is that a large fraction of them shows a fully concordant classification: $85.5 \%$ $(84.4 \%)$ of the analyzed galaxies in the high-quality (full) sample can be assigned consistently to the red or blue branch of the bimodal distribution using spectral, photometric, and morphological criteria. Due to the redshift constraints imposed by the spectral classification, we cannot explore a very large redshift domain. However, we divided the useful redshift range into two bins, so that each bin contained a similar number of objects. In the low- $z$ range $(0.45 \leq z \leq 0.73), 83.4 \%$ of the galaxies show a fully concordant classification, while in the high-redshift range $(0.73 \leq z \leq 1.25)$ this percentage slightly grows to $85.5 \%$. We conclude that the fraction of galaxies for which the three classification schemes agree remains constant (within our statistical errors) for the entire redshift range covered by our analysis.

\section{Conclusion}

We have presented a classification scheme for galaxies observed by the $1 k$ zCOSMOS-bright sample, developed using spectral, color, and morphological information. Since the spectral classification that we adopted is based on measurements of the [OII] and D4000 features, and given the wavelength range of 


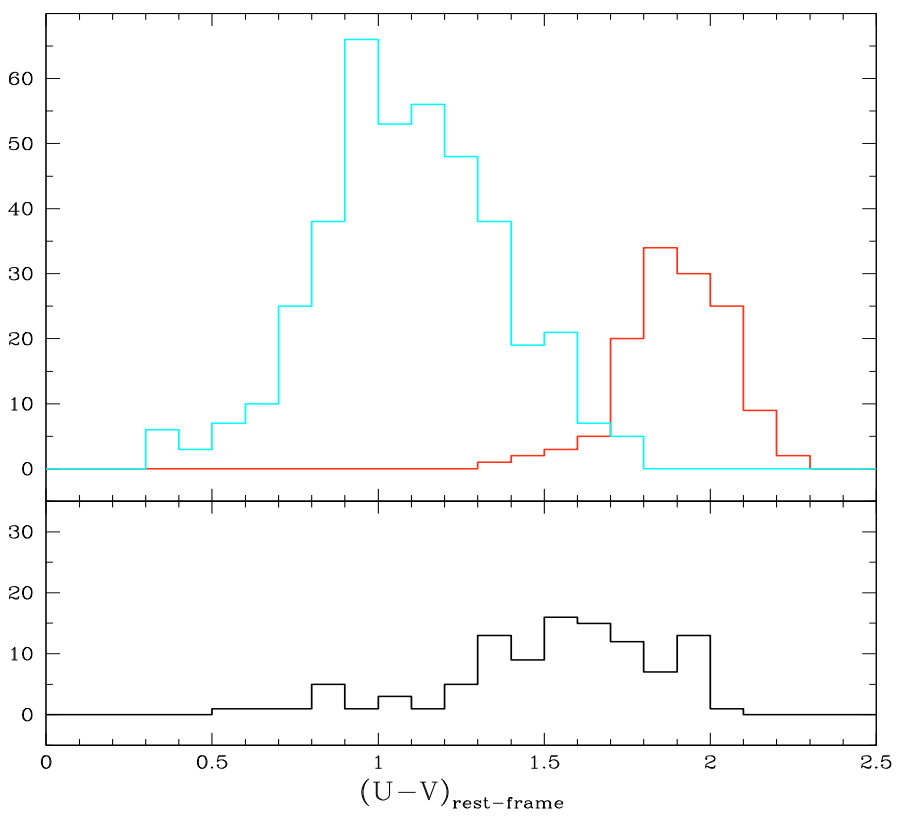

Fig. 10. Classification cube and rest-frame $U-V$ color of the $1 k$ zCOSMOS-bright data set. The upper panel shows the rest-frame color distributions of galaxies with fully concordant classification, the blue histogram representing the star-forming, blue, disk-dominated galaxies, while the red histogram is for the quiescent, red, bulgedominated galaxies. The lower panel shows the rest-frame color distribution for the outliers, i.e. galaxies that do not present the same behavior in all the three classifications.

the zCOSMOS spectra, this classification scheme has been applied to galaxies in the redshift range $0.45 \leq z \leq 1.25$. We have demonstrated that the adopted spectral classification is an effective way of separating galaxies into two main classes, due to the high quality of the zCOSMOS data.

We have found that (i) an excellent correlation exists between the classification from spectral data (quiescent/starforming galaxies) and the one based on colors (red/blue galaxies), and (ii) a similarly good correlation exists between the classification based on spectral data and the one based on morphological analysis (early-/late-type galaxies) only if we complement the morphological classification with additional color information. The "outliers" in this simple 2-way analysis of different classification methods (i.e. spectra versus colors and spectra versus morphology) are quiescent galaxies (high $D 4000$ value) with emission lines (about $4 \%$ of the total sample of galaxies) and morphologically intermediate galaxies (about $20 \%$ of the total sample of galaxies) that consists of both blue/star-forming and red/quiescent galaxies. These are the morphologically-classified galaxies that require additional color information to ensure good agreement between the classification of schemes using both spectroscopic and morphological data.

Finally, analyzing the distribution of all galaxies in a 3D-classification cube, based on the three different classification methods, we have found that for about $85 \%$ of the galaxies there is complete agreement between the classifications (i.e. quiescent, red, bulge-dominated galaxies $(\sim 20 \%)$ or star-forming, blue, disk-dominated galaxies $(\sim 65 \%)$ ). If additional color information was not used for the ZEST-type $=2.1$ galaxies (i.e. by assigning all of them to the spheroid- or to the disk- galaxies), the percentage of fully concordant classifications would decrease to about $75 \%$.
The correspondence between the classification cube and classical representation of galaxy bimodality, which uses the rest-frame $U-V$ colors $^{4}$, is shown in Fig. 10. In the upper panel, as expected, the rest-frame $U-V$ color distributions of the two galaxy groups with fully concordant classifications are separated well and reproduce the bimodality. Perhaps more interesting is the distribution of outliers in the lower panel, which is centered between the two branches of the galaxy bimodality, in the socalled "green valley", the relatively sparse region between the blue cloud of star-forming galaxies and the red sequence of quiescent ones.

The results presented in this paper demonstrate the potential of the data set provided by the COSMOS/zCOSMOS survey for investigating the nature of the observed relationships between galaxy properties and highlight fundamental aspects of the galaxy population. The main conclusion of this work is that the bimodality is a prevalent feature of the galaxy population, at least out to $z \sim 1$, in three critical parameters describing galaxy physical properties: morphological, color, and spectroscopic diagnostic line data. More detailed analysis of the properties of both a wider sample of galaxies and outliers in this 3D-classification cube, based on an analysis of the larger $10 \mathrm{~K}$ zCOSMOS-bright sample, will be presented elsewhere.

Acknowledgements. This work was partially supported by INAF under PRIN-2006/1.06.10.08 and by ASI under grant ASI/COFIS I/016/07/0.

\section{References}

Bailin, J., \& Harris, W. E. 2008, ApJ, 681, 225

Baldwin, J. A., Phillips, M. M., \& Terlevich, R. 1981, PASP, 93, 5

Balogh, M. L., Eke, V., Miller, C., et al. 2004a, MNRAS, 348, 1355

Balogh, M. L., Baldry, I. K., Nichol, R., et al. 2004b, ApJ, 615, L101

Baldry, I. K., Glazebrook, K., Brinkmann, J., et al. 2004, ApJ, 600, 681

Ball, N. M., Loveday, J., Brunner, R. J., et al. 2006, MNRAS, 373, 845

Bell, E. F., Wolf, C., Meisenheimer, K., et al. 2004, ApJ, 608, 752

Bershady, M. A., Jangren, A., \& Conselice, C. 2000, AJ, 119, 2645

Bertin, E., \& Arnouts, S. 1996, A\&AS, 117, 393

Brinchmann, J., Charlot, S., White, S. D. M., et al. 2004, MNRAS, 351, 1151

Brusa, M., Zamorani, G., Comastri, A., et al. 2007, ApJS, 172, 353

Bruzual, G. 1983, ApJ, 273, 105

Capak, P., Mobasher, B., Abraham, B., et al. 2007, ApJS, 172, 99

Cassata, P., Cimatti, A., Kurk, J., et al. 2008, A\&A, 483, L39

Cimatti, A., Mignoli, M., Daddi, E., et al. 2002, A\&A, 392, 395

Cirasuolo, M., McLure, R. J., Dunlop, J. S., et al. 2007, MNRAS, 380, 585

Coleman, G. D., Wu, C.-C., \& Weedman, D. W. 1980, ApJS, 43, 393

Colless, M., Ellis, R. S., Taylor, K., \& Hook, R. N. 1990, MNRAS, 244, 408

Conselice, C. J. 2006, MNRAS, 373, 1389

Drory, N., \& Fisher, D. B. 2007, ApJ, 664, 640

Feldmann, R., Carollo, C. M., Porciani, C., et al. 2006, MNRAS, 372, 565

Fioc, M., \& Rocca-Volmerange, B. 1999, A\&A, 351, 869

Forster, K., Green, P. J., Aldcroft, T. L., et al. 2001, ApJS, 134, 35

Franzetti, P., Scodeggio, M., Garilli, B., et al. 2007, A\&A, 465, 711

Fukugita, M., Shimasaku, K., \& Ichikawa, T. 1995, PASP, 107, 945

Giallongo, E., Salimbeni, S., Menci, N., et al. 2005, ApJ, 622, 116

Ilbert, O., Tresse, L., Zucca, E., et al. 2005, A\&A, 439, 863

Hubble, E. 1938, PASP, 50, 97

Humason, M. L. 1936, ApJ, 83, 10

Kauffmann, G., Heckman, T. M., White, S. D. M., et al. 2003, MNRAS, 341, 33 Kennicutt, R. C. 1992, ApJS, 79, 255

Le Fèvre, O., Vettolani, G., Garilli, B., et al. 2005, A\&A, 439, 845

Lamareille, F., Contini, T., Le Borgne, J.-F., et al. 2006, A\&A, 448, 893

Lilly, S. J., Le Févre, O., Crampton, D., et al. 1995, ApJ, 455, 50

Lilly, S. J., Le Fèvre, O., Renzini, A., et al. 2007, ApJS, 172, 70

Manning, C. V. 2002, ApJ, 574, 599

Mignoli, M., Cimatti, A., Zamorani, G., et al. 2005, A\&A, 437, 883

Sargent, M. T., Carollo, C. M., Lilly, S. J., et al. 2007, ApJS, 172, 434

\footnotetext{
4 The rest-frame colors were computed following the method developed by Ilbert et al. (2005).
} 
Sawicki, J. J., Lin, H., \& Yee, H. K. C. 1997, AJ, 310, 1

Scarlata, C., Carollo, C. M., Lilly, S. J., et al. 2007a, ApJS, 172, 406 Scarlata, C., Carollo, C. M., Lilly, S. J., et al. 2007b, ApJS, 172, 494 Scodeggio, M., Franzetti, P., Garilli, B., et al. 2005, PASP, 117, 1284 Scoville, N., Abraham, R. G., Aussel, H., et al. 2007, ApJS, 172, 38 Strateva, I., Ivezić, Z., Knapp, G. R., et al. 2001, AJ, 122, 1861 Taniguchi, Y., Scoville, N., Murayama, T., et al. 2007, ApJS, 172, 9 van den Bergh, S. 2007, AJ, 134, 1508

Wild, V., Peacock, J. A., Lahav, O., et al. 2005, MNRAS, 356, 247

Yan, R., Newman, J. A., Faber, S. M., et al. 2006, ApJ, 648, 281

1 INAF - Osservatorio Astronomico di Bologna, Bologna, Italy e-mail: marco.mignoli@oabo.inaf.it

2 INAF - Istituto di Astrofisica Spaziale e Fisica Cosmica, Milano, Italy

3 Dipartimento di Astronomia, Università degli Studi di Bologna, Bologna, Italy
${ }^{4}$ INAF - Osservatorio Astronomico di Arcetri, Firenze, Italy Institute of Astronomy, ETH Zurich, Zurich, Switzerland

${ }^{6}$ Laboratoire d'Astrophysique de Toulouse-Tarbes, Université de Toulouse, CNRS Toulouse, France

7 Laboratoire d'Astrophysique de Marseille, Marseille, France

8 European Southern Observatory, Garching, Germany

9 Dipartimento di Astronomia, Università di Padova, Padova, Italy

${ }^{10}$ Max Planck Institut für Extraterrestrische Physik, Garching, Germany

11 INAF - Osservatorio Astronomico di Brera, Milano, Italy

12 California Institute of Technology, Pasadena, CA, USA

13 Centre de Physique Theorique, Marseille, Marseille, France

14 Institut d'Astrophysique de Paris, Université Pierre \& Marie Curie, Paris, France

15 INAF - Osservatorio Astronomico di Roma, Monte Porzio Catone, Italy 